\title{
The Bending Energy of a Semi-Flexible Polymer Chain and the Polygons of the Polymer Chain
}

\author{
Pramod Kumar Mishra \\ Department of Physics, \\ DSB Campus, Kumaun University, \\ Nainital (Uttarakhand) India-263002
}

\begin{abstract}
We consider random walk model for a semiflexible polymer chain using a square and the cubic lattices to enumerate conformations of the polymer chain in two and three dimensions, respectively. The bending energy of the chain is assumed as the key factor which controls the minimum average length of the chain in between two successive bends in the chain; and the average length of the chain in between its two successive bends is defined as the persistence length $\left(l_{p}\right)$ of the polymer chain. Our analytical estimate suggests that the minimum energy required to bend the chain is $\mathrm{E}_{b}=\mathrm{k}_{\mathrm{B}} * \mathrm{~T}^{*} \log [2 *(\mathrm{~d}-$ 1) $* g * l_{p}$ ], (where $d, g$ and $l_{p}$ represents the dimensionality of the space, the step fugacity of the chain and the persistence length of the polymer chain, respectively), which is required to bend the chain so that a polymer loop of the perimeter $4 * l_{p}$ may be formed. Our estimate of the bending energy is independent of the fact that whether chain is ideal or the self- avoiding polymer; where in the case of ideal chain the vortex of the chain is treated as a monomer (the vertex version) while in the case of the selfavoiding polymer model each bond of the walk is treated as a monomer of the polymer chain (the bond version). The method of calculations of thermodynamics of the chain may be easily extended to the case of isotropic walk polymer model, the partially and the fully directed walk models of the polymer chain.
\end{abstract}

Keywords - Semi-flexible polymer; polymer polygon; bending energy; persistence length;

\section{INTRODUCTION}

The macromolecules play vital role in the living creatures and such molecules are also found in the non living things. In the case of the living creature the DNA and the Proteins are the useful macromolecules. The DNA and the Proteins are known for their excellent elastic properties. These Bio-molecules are the soft objects and therefore they may be easily confined to fairly small volume or the space. These molecules are known to have variable range of stiffness or the elastic properties. The macromolecule which requires no energy to introduce bend in its segments is known as the flexible macromolecule, however, those macromolecules which require very large amount of the bending energy to produce even a single bend in its conformations are known as the stiff macro-molecules. Thus, the molecules which require intermediate value of the bending energy to produce bends in its segments, and thus these molecules are known as the semi-flexible macromolecules. Such molecules are important and key to Biological activities (replications, gene regulations, etc.) occurring in the living creatures [1-2].
The process of replication of the DNA or the Proteins is the useful factor to the Biological actions which are occurring in the living cells. The process like the replication or the gene regulations may take place easily provided the macromolecule has conformation like a loop (polygon). Therefore, the formation of the loop in an amino acid chain is one of the key in the Protein folding events. Thus, the possibility of the formation of the polymer loop (polygon) may be an interesting aspect which has been considered in this report. The Biological macromolecules are heterogeneous and also the distribution of the different types of the monomers (repeat units) along the back bone of the chain is also random [3-8].

However, in this report we consider the model system where we consider a linear homogenous polymer chain to describe the possibility of the formation of the polymer polygon on the basis of simple analytical calculations. We relied to the analytical methods of the recursion relations [8-10] to calculate the partition function of the semi-flexible polymer chain in the two and three dimensions and also to estimate the value of the bending energy required to form a polymer loop of the perimeter $4 * l_{p}$ and the polygon area $1_{p}^{2}$.

This manuscript is organized as follows: In the sec.-II, we outline the polymer model for an ideal chain in two as well as three dimensions. Since, we consider the polymer polygon which has close loop like structure and therefore, we consider the polymer chain as an ideal polymer chain. The method of the recursion relations were discussed in brief in the section III, which is used to calculate the partition function of the polymer chain in two as well as three dimensions, respectively. In the section III, we calculate the persistent length [7-13] of the polymer chain and we also derive an expression for the bending energy of the chain conformations which are having shape like a loop (the polygon). A brief discussion on the bending energy which is required to form polygon of required size is obtained, and we conclude the discussion in the section IV by summarizing our findings.

\section{THE MODEL}

The random walk lattice model [7-15] is used on a square and a cubic lattice to mimic the polymer chain conformations. The walker is assumed to begin stepping from a point (i. e. O; a point where one end of the chain is grafted), and the walker takes steps along all possible directions to mimic the polymer chain conformations and only those conformations were 
taken into considerations which have the end monomers on the same site where the polymer chain is assumed to be grafted. In this case, since the close loops (polygons, as schematically shown in Fig. 1) of the chain is considered therefore the chain is modeled like an ideal polymer. The walker, thus can take steps along $\pm \mathrm{x}$ and $\pm \mathrm{y}$ directions in the case of the square lattice (i. e. in the case of two dimensional model) while in the case of

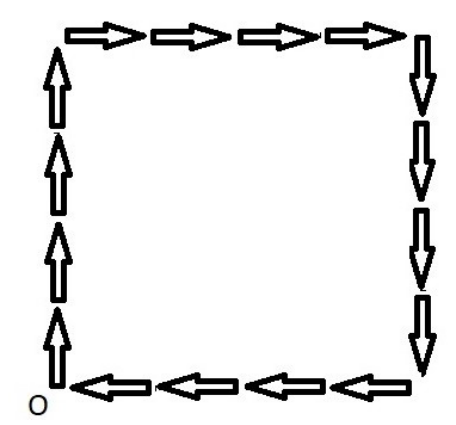

Fig. 1: We have shown the polymer polygon schematically. In this figure, each arm of the polygon of the polymer has $l_{p}(=4)$ monomers. Thus the perimeter of the polygon is $4 * l_{p}$ while area of the polymer polygon is $l_{p} * l_{p}$.

the cubic lattice the walker may take steps along $\pm \mathrm{x}, \pm \mathrm{y}$ and $\pm \mathrm{z}$ directions, when the chain conformations are considered in three dimensions [7-10].

However, in the case of self-avoiding walk model we may choose bond of the walk as a monomer to mimic real polymer conformations and even then one may obtain polymer polygon where there is no possibility of the overlap of monomers or to occupy same site by any of the monomer.

The grand canonical partition function $[G(g, k)]$ of the polymer chain may be written in general as [7-11],

$$
G(g, k)=\sum_{N=0}^{\infty} \sum_{N_{B}=0}^{N-1} g^{N} k^{N_{B}}
$$

Where, $g$ is the step fugacity of the monomer, $k$ [=exp($\left.E_{b} / k_{B} T\right)$ ] is Boltzmann weight corresponding to bending energy of the chain, an $N$ is the number of monomers in the chain and $N_{B}$ is the number of bends in the chain of the $N$ monomers. The term $k_{B} T$ is the thermal energy at the temperature $T$ and $k_{B}$ is the Boltzmann constant. The persistence length $\left(l_{p}\right)$ [10-13] of the polymer chain may be defined as the average length of the polymer chain for per unit bend in the chain, and it may be calculated from the following relations: $1_{p}=\left\langle N>/<N_{B}>\right.$

$$
=\quad \frac{\partial \log (\mathrm{G}(\mathrm{g}, \mathrm{k}))}{\partial \log (\mathrm{g})} / \frac{\partial \log (\mathrm{G}(\mathrm{g}, \mathrm{k}))}{\partial \log (\mathrm{k})}
$$

It is also to be noted here that we consider those conformations of the polymer chain which has the starting and the end monomers at the site where the polymer chain is assumed to be grafted. The perimeter of the polymer polygon is $4^{*} n$ monomers (where $\mathrm{n}=\mathrm{l}_{\mathrm{p}}$ ) i. e. the $n$ monomers are along each of the direction so that the polymer polygon may be closed one, where $n=1,2,3 \ldots, l_{p}$ monomers.

\section{METHOD OF CALCULATIONS}

The recursion relations for the square lattice may be written in the following manner [10-17];

$$
\begin{aligned}
& \left(1-x_{1}\right) * X_{1}-x_{1} * K^{*} X_{2}-x_{1} * k * Y_{1}-x_{1} * k * Y_{2}=x_{1} \\
& -x_{2} * K^{*} X_{1}+\left(1-x_{2}\right) * X_{2}-x_{2} * k * Y_{1}-x_{2} * k * Y_{2}=x_{2} \\
& -y_{1} * k * X_{1}-y_{1} * k * X_{2}+\left(1-y_{1}\right) * Y_{1}-y_{1} * K^{*} Y_{2}=y_{1} \\
& -y_{2} * k * X_{1}-y_{2} * k * X_{2}-y_{2} * K^{*} Y_{1}+\left(1-y_{2}\right) * Y_{2}=y_{2}
\end{aligned}
$$

And for the cubic lattice case the recursion relations may be written as [10-17];

$\left(1-x_{1}\right) * X_{1}-x_{1} * K * X_{2}-x_{1} * k * Y_{1}-x_{1} * k * Y_{2}-x_{1} * k * Z_{1}-x_{1} * k * Z_{2}=x_{1}$

$-x_{2} * K * X_{1}+\left(1-x_{2}\right) * X_{2}-x_{2} * k * Y_{1}-x_{2} * k * Y_{2}-x_{2} * k * Z_{1}-x_{2} * k * Z_{2}=x_{2}$

$-y_{1} * k * X_{1}-y_{1} * k * X_{2}+\left(1-y_{1}\right) * Y_{1}-y_{1} * K * Y_{2}-y_{1} * k * Z_{1}-y_{1} * k * Z_{2}=y_{1}$

$-y_{2} * k * X_{1}-y_{2} * k * X_{2}-y_{2} * K * Y_{1}+\left(1-y_{2}\right) * Y_{2}-y_{2} * k * Z_{1}-y_{2} * k * Z_{2}=y_{2}$

$-z_{1} * k * X_{1}-z_{1} * k * X_{2}-z_{1} * k * Y_{1}-z_{1} * k * Y_{2}+\left(1-z_{1}\right) * Z_{1}-z_{1} * K * Z_{2}=z_{1}$

$-z_{2} * k * X_{1}-z_{2} * k * X_{2}-z_{2} * k * Y_{1}-z_{2} * k * Y_{2}-z_{2} * K * Z_{1}+\left(1-z_{2}\right) * Z_{2}=z_{2}$

Where $X_{l}$ is the sum of the Boltzmann weight of all the walks with first step along $+x$ direction, while $X_{2}$ is the Boltzmann weight of all walks with first step along $-x$ direction, and similarly we may define $Y_{1}, Y_{2}, Z_{1}$ and $Z_{2}$ as the sum of Boltzmann weights corresponding to the first step along $+y$, $y,+z$ and $-z$ directions, respectively. The $x_{1}, x_{2}, y_{1}, y_{2}, z_{1}$ and $z_{2}$ are the step fugacity of the steps (monomers) along $+x,-x$, $+y,-y,+z$ and $-z$ directions, respectively. The parameter $k$ is the Boltzmann weight corresponding to bending angle $90^{0}$ and while the $K$ is the Boltzmann weight corresponding to the bending angle $180^{\circ}$.

For the sake of simplicity, all steps may be represented with the step fugacity equal to $g$.

We may solve set of four equations shown by equation eqn. no. (3) to get value of $X_{1}, X_{2}, Y_{1}$ and $Y_{2}$, for two dimensional model and thus in this case we have partition function is written as- $G^{2 D}(g, k)=X_{1}+X_{2}+Y_{1}+Y_{2}$; and similarly by solving another set of six equations as show in the equation no. 4 , as in the case of three dimensional model to get the values of $X_{l}$, $X_{2}, Y_{1}, Y_{2}, Z_{1}$ and $Z_{2}$. The partition function for the cubic lattice case may be obtained as $G^{3 D}(g, k)=X_{1}+X_{2}+Y_{1}+Y_{2}+Z_{1}+Z_{2}$.

We calculate the average length of the polymer chain and also the average number of the bends in the chain so that we may have value of the persistence length $\left(l_{p}\right)$ of the polymer chain using equation no. (2). It has been found from these calculations that the bending energy of the chain conformations in two dimensions may vary with polygon size $\left(n=l_{p}\right)$ as,

$$
E_{b}=k_{B} * T * \log \left[2 * g * l_{p}\right]
$$


While, in the case of three dimensions or for the case of a cubic lattice the bending energy of the polymer chain may be expressed as (as shown in fig. 2),

$$
E_{b}=k_{B} * T * \log \left[4 * g * l_{p}\right]
$$

\section{THE RESULTS AND THE CONCLUSIONS}

The random walk lattice model is used to mimic the conformations of a long linear semi-flexible polymer chain in two and three dimensions using the square and the cubic lattices, respectively. One end of the polymer chain is grafted

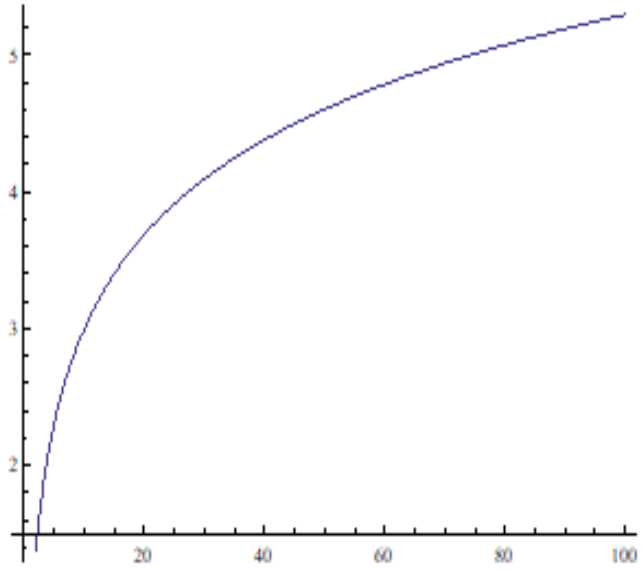

$\log \left[2 * \mathrm{~g}^{*} \mathrm{l}_{p}\right]$

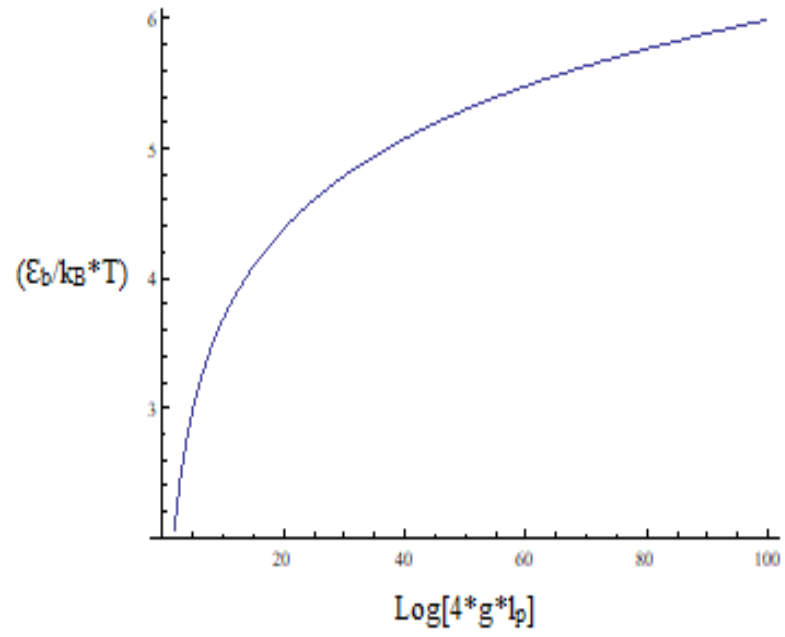

Fig. 2: The variation of $\left(E_{b} / k_{B} * T\right)$ versus $\log \left[2 * g * l_{p}\right]$ is shown for two dimensional case, while the variation of $\left(E_{b} / k_{B} * T\right)$ versus Log[4*g*l $\left.l_{p}\right]$ is also shown for the case of three dimensions in this figure (here $\mathrm{g}=1$ unit).

on a point $(\mathrm{O})$ and all those conformations of the polymer chain were considered which have starting as well as the end monomers on the site where the polymer chain is grafted. It has been found that there are 8 polygons (each polygon has the perimeter $4 * l_{p}$ and the area is $l_{p}^{2}$ ) in the case of two dimensions and while there are 12 polygons (each polygon has the perimeter $4 * l_{p}$ and the area is $l_{p}^{2}$ ) which are found to have first as well as the last monomer on the site $(\mathrm{O})$ where the polymer chain is grafted. The probability of finding such polymer polygon in two dimensions is $8 / 4^{P}$ and in three dimensions the probability is $12 / 6^{P}$ (where $P=4 * l_{p}$, i. e. perimeter of the polygon). The value of $l_{p}$ is $1,2,3, \ldots \ldots, n$ monomers.

The standard methods of the statistical Physics were used to calculate the persistence length of the polymer chain. It has been found in the case of two as well as three dimensions that the bending energy of the chain may be expressed as the function of polygon size $\left(l_{p}\right)$ as,

$E_{b}=k_{B} * T * \log \left[2 *(d-1) * g * l_{p}\right]$

Thus, in order to summarize, the random walk lattice model has been used on square and cubic lattices to calculate the bending energy of the polymer chain and the bending energy which is required to form polymer polygon (loop) of the perimeter size $4 * l_{p}$ is shown using eqn. no. 7 . The bending energy of the chain varies for two and three dimensions and the variation of the bending energy is shown using fig. 2 . It is also to be noted here that the results expressed through equation nos. (3) \& (4) are independent of the fact that the polymer chain is either ideal chain (vertex version; each vertex of the step is treated as a monomer) or the self avoiding chain (bond version; each bond/edge of the walker is treated as a monomer).

The method described above may be easily extended to another case of polymer models. For example, by substitution of $x_{2}=0$ and $y_{2}=0$ in above equation no. (3), we may obtain recursion relations for the fully directed walk model of the two dimensional polymer chain and also accordingly the partition function of the fully directed chain may be obtained, while substituting only either $x_{2}=0$ or $y_{2}=0$ in the equation no. 3 , we may find the partition function of the partially directed polymer chain in two dimensions. Similarly by substitution of $x_{2}=0, y_{2}=0$ and $z_{2}=0$, we may obtain the partition function of the fully directed polymer chain in three dimensions. Thus, using above discussed method we may discuss thermodynamics of the polymer chain for different versions of the partially and fully directed walk models of the polymer chain in two and three dimensions.

About the Author-Dr. P. K. Mishra is serving to Kumaun University since 19.03.2005 as a regular faculty and he 
published several articles as a sole author in the journals of international repute;

\section{REFERENCES}

[1] W. Huang and V. Zaburdaev, "The shape of pinned forced polymer loops", Soft Matter, vol. 15, pp. 1785-1792, 2019.

[2] N. M. Toan, G. Morrison, C. Hyeon and D. Thirumalai, "Kinetics of loop formation in polymer chain', J. Chem. Phys. B, vol. 112, pp. 6094, 2008

[3] S. Jun and J. Bechhoefer, "Role of polymer loops in DNA replication', Physics in Canada, vol. 59, pp. 85-92, 2003.

[4] S. Tyagi, S. A. E. Marris and F. R. Kramer, "Wavelength-shifting molecular beacons", Nature Biotech., vol. 18, pp. 1191, 2000.

[5] R. Schleif, "DNA looping", Annu. Rev. Biochem. Sci., vol. 26 , pp. 199, 1992.

[6] K. Rippe, "Making contacts on a nucleic acid polymer", Trends Biochem. Sci., vol. 26, pp. 733, 2001.

[7] O. Kratky, G. Porod, "Recl. Trav. Chim. Pays-Bas", vol. 68, pp. 1160,1949

[8] V. Privman, N.M. Svrakic, "Directed Models of Polymers, Interfaces and Clusters: Scaling and Finite-Size Properties", Springer, Berlin, 1989.

[9] M. Doi, S.F. Edwards, "Theory of Polymer Dynamics", Clarendon, Oxford, 1992
[10] P. K. Mishra, S. Kumar and Y. Singh,“A simple and exactly solvable model for a semiflexible polymer chain interacting with a surface', Physica A, vol. 323, pp. 453-465, 2003.

[11] J. Zi Zhang, X. Y. Peng, S. Liu, B. P. Jiang, S. C. Ji and X. C. Shen, "The persistence length of semiflexible polymers in lattice Monte Carlo simulations", Polymers, vol. 11, pp. 295, 2019.

[12] P. K. Mishra, "A semiflexible alternating copolymer chain adsorption on a flat and a fluctuating surface", J Phys: Cond Matt., vol. 22, pp. 155103, 2010.

[13] P. K. Mishra, "Divergence of persistent length of a semiflexible homopolymer chian in the stiff chain limit', Fizika A, vol. 19, pp. 145-152, 2010

[14] P. K. Mishra, "Effect of geometrical constraint on conformational properties of a polymer chain', Phase Transitions, vol. 84, pp 291-298, 2011.

[15] P. K. Mishra, "A semiflexible polymer chain under geometrical restrictions: Only bulk behaviour and no surface adsorption", Condens Matter Phys., vol. 17, pp. 23001, 2014.

[16] P. K. Mishra, "Equilibrium statistics of an infinitely long chain in the severe confined geometry: exact results", Phase Transitions, vol. 88 pp. 593-604, 2015.

[17] P. K. Mishra, "Effect of confinement and stiffness on the conformational changes of a semiflxible homopolymer chain", Ind J Phys., vol. 91 pp. 1297-1304, 2017. 